\title{
Análise dos Indicadores de Desempenho do Programa Nacional de Melhoria do Acesso e da Qualidade da Atenção Básica (PMAQ-AB) no Nordeste Brasileiro
}

\section{Analysis of the Performance Indicators of the National Program for Improvement in Access and Quality of Primary Care (PMAQ-AB) in Northeast Brazil}

\section{RESUMO}

Objetivo: Avaliar os indicadores de desempenho das equipes de saúde da família (eSF) participantes do $1^{\circ}$ e $2^{\circ}$ ciclo do PMAQ/AB no Nordeste brasileiro. Metodologia: Trata-se de um estudo ecológico, realizado a partir de dados referentes aos indicadores de saúde registrados por 4.325 eSF no Sistema de Informação à Saúde para a Atenção Básica (SISAB) de acesso restrito e solicitados ao Ministério da Saúde. Os dados foram categorizados em 24 indicadores de desempenho, utilizados para a classificação das eSF participantes do PMAQ-AB, no $1^{\circ}$ ciclo (2012) e $2^{\circ}$ ciclo (2014). Resultados: Do total de indicadores, 17 do $2^{\circ}$ ciclo alcançaram valores inferiores ao $1^{\circ}$ ciclo. Dos cinco indicadores com melhor desempenho no $2^{\circ}$ ciclo, apenas três apresentaram diferenças significativas $(p<0,001)$, sendo eles a proporção de pacientes diabéticos cadastrados, a média de consultas médicas/habitante, a proporção de consultas médicas para cuidado médico continuado/prolongado. Os indicadores relativos à saúde da mulher e da criança foram os que apresentaram os menores valores, ficando abaixo do $1^{\circ}$ ciclo $(p<0,001)$. Ocorreram variações entre os indicadores dos Estados do Nordeste $(p<0,05)$, sendo este efeito observado principalmente no Maranhão, Alagoas e Piauí. Conclusão: A maioria dos indicadores apresentou menor desempenho no $2^{\circ}$ Ciclo do PMAQ/AB, demonstrando pouca melhoria na qualidade e no acesso aos serviços ofertados pela $A B$, especialmente àqueles direcionados às mulheres e as crianças do Nordeste.

\section{DESCRITORES}

Avaliação em Saúde. Atenção Primária à Saúde. Indicadores Básicos de Saúde. Estratégia Saúde da Família.

\begin{abstract}
Objective: To analyze the performance indicators of the eSF teams that participated in the 1st and 2nd cycle of the PMAQ/ $A B$ in northeastern Brazil. Methodology: This is an ecological study, based on data on health indicators registered by eSF in the restricted access Health Information System (SISAB), and requested by the Ministry of Health. The indicators were categorized in 24 performance indicators, used to classify the eSF participants in the PMAQ-AB, in the 1st cycle (2012) and the 2 nd cycle (2014). Results: Of the total indicators, 17 of the 2 nd cycle reached values lower than the 1 st cycle. Of the 7 best performing indicators in the 2 nd cycle, only three presented significant differences $(p<0.001)$, which were the proportion of diabetic patients registered, average of medical/usual visits, medical consultation rates for continued/prolonged medical treatment. The indicators related to the health of women and children presented the lowest values, being under the 1 st cycle $(p<0.001)$. Conclusion: Most indicators underperformed the 2 nd Cycle of the PMAQ/AB, showing little improvement in quality and access to services offered by the $A B$, especially those directed for women and children from the northeast.
\end{abstract}

\section{DESCRIPTORS}

Health Assessment. Primary Health Care. Basic Health Indicators. Family Health Strategy.

${ }^{1}$ Departamento de Odontologia Social, Faculdade de Odontologia de Piracicaba, Universidade Estadual de Campinas. Piracicaba, São Paulo, Brasil. 
A Atenção Básica $(A B)$ reforçou a proposta de reorientação de um modelo ideológico assistencial da atenção à saúde, anteriormente centrada na assistência médica e individual, para um modelo com enfoque na vigilância em saúde centrado nas equipes de saúde e na população. Assim, a AB passou a coordenar uma rede de serviços pautadas nos princípios e diretrizes do Sistema Único de Saúde (SUS) e implementada por meio da Estratégia Saúde da Família (ESF). Nesse contexto, a AB reflete uma visão diferenciada no processo de prestação de serviços, incluindo ações de saúde individuais, familiares e coletivas que envolvem todas as linhas de cuidado, sob a responsabilidade sanitária de uma equipe multiprofissional em um território adscrito" .

Para a organização dessas ações se faz necessária a avaliação constante dos serviços ofertados à comunidade pelas equipes de saúde da família (eSF)2. Isso porque, a avaliação é um dispositivo que pode contribuir para o aumento da capacidade de analisar e intervir em determinadas situações, contribuindo para tomada de decisão respaldada em evidências com o intuito de diminuir as adversidades em saúde e atender, com mais eficiência, as diversas demandas e necessidades da população ${ }^{3}$.

Historicamente, o processo de avaliação da Atenção Básica no Brasil é recente, perpassando pela Avaliação da Melhoria da Qualidade (AMQ) em 2005, e aprimorada em 2011, com o Programa Nacional de Melhoria do Acesso e da Qualidade da Atenção Básica (PMAQ/AB). O PMAQ/AB tinha como objetivo principal a indução da ampliação do acesso e da melhoria da qualidade da $A B$, além de contribuir financeiramente com os municípios que atingissem os padrões de qualidade estabelecidos pelo programa ${ }^{4}$

Para o alcance dessa finalidade, o $P M A Q / A B$ constituiu-se de quatro fases: adesão e contratualização, desenvolvimento, avaliação externa e recontratualização. Durante a fase de desenvolvimento, o PMAQ/ $A B$ selecionava indicadores de saúde para a avaliação do desempenho das equipes, que tinham referência com alguns dos principais focos estratégicos da Atenção Básica (prénatal, prevenção do câncer do colo de útero, saúde da criança, controle da hipertensão arterial sistêmica e do diabetes mellitus, saúde bucal, saúde mental, doenças transmissíveis e produção geral). Esses indicadores também guardavam relação com os indicadores pactuados no âmbito do Pacto Pela Saúde e estabelecidos nas três esferas de governo ${ }^{5}$.

Considerando o escopo do programa e os seus mecanismos propostos para o desenvolvimento da qualidade da atuação das eSF, diversos estudos foram realizados para mensurar seus efeitos no cotidiano da $A B^{6-8}$. Em se tratando da Região Nordeste do Brasil, observada como uma das regiões que mais apresentam problemas de saúde pública, os estudos ainda são escassos. Nessa região pesquisas foram desenvolvidas para a identificação da satisfação dos usuários durante o programa ${ }^{7}$, compreensão do aumento de acesso à atenção primária ${ }^{8}$ e aos serviços odontológicos $^{9}$, análise da incorporação da autoavaliação pelas equipes ${ }^{10}$, avaliação da estrutura, insumos e atividades integradas 
com outros níveis de atenção ${ }^{11}$, no entanto, não ocorreu ainda, uma avaliação para identificar o desempenho dos indicadores de saúde da população assistida pelas eSF, ao longo dos ciclos do PMAQ-AB.

Assim, para compreender este cenário e contribuir com apontamentos para o aprimoramento do processo de trabalho da ESF, o presente estudo propôs avaliar os indicadores de saúde, conhecidos como indicadores de desempenho, registradas pelas eSF participantes do $1^{\circ}$ e $2^{\circ}$ ciclo do PMAQ/ AB no Nordeste brasileiro.

\section{METODOLOGIA}

Trata-se de um estudo ecológico, o qual obteve dispensa do Comitê de Ética e Pesquisa da FOP-UNICAMP por se tratar de uma pesquisa envolvendo dados secundários.

Os dados do estudo são referentes aos indicadores de saúde do $1^{\circ}$ e $2^{\circ}$ Ciclo do programa, referente aos anos de 2012 e 2014, respectivamente, e foram solicitados ao Ministério da Saúde no ano de 2016.

Os dados são armazenados no banco de base nacional de monitoramento e avaliação dos indicadores de saúde do PMAQ-AB, organizado pelo Departamento de Atenção Básica (DAB). O arquivo enviado pelo Ministério da Saúde aos pesquisadores, continha 49.918 registros de todas as regiões brasileiras e unidades de saúde participantes nos dois ciclos, sendo 17.482 no ciclo $1^{\circ}$ e 32.436 no ciclo $2^{\circ}$. Deste total foram excluídos 12.491 registros devido à ausência de eSF e/ou equipe de Saúde Bucal (eSB) ou por ser Núcleo de
Apoio à Saúde da Família (NASF) restando 37.427 registros. Dos 37.437 registros foram identificadas 10.882 eSF que constavam nos dois ciclos com todos os dados de identificação iguais nos dois ciclos, deste total 4.325 eram da Região Nordeste. Segundo o Ministério da Saúde, no final do ano de 2014, havia 18.215 equipes de saúde credenciadas no Nordeste ${ }^{12}$, assim, o presente estudo obteve dados de $23,74 \%$ da cobertura da AB nesta região do país.

Destaca-se que o PMAB/AB utiliza a seguinte classificação frente aos indicadores de saúde: Indicadores de desempenho e de monitoramento. Os indicadores classificados como de desempenho são aqueles que foram analisados e fizeram parte da composição da nota no momento da avaliação. Os indicadores de monitoramento são aqueles estimulados pelo programa para serem acompanhados pela a equipe, e são registrados no Sistema de Informação da Atenção Básica (SISAB). O SISAB, integra dois sistemas de softwares, responsáveis por armazenar informações, que são registrados por todos os profissionais da ESF. As informações são sobre a produção da equipe, situação sociossanitária e de saúde da população do território ${ }^{13}$. Esse sistema foi utilizado pelo Ministério da Saúde para a coleta dos indicadores de desempenho e de monitoramento, uma vez que esse era o único sistema disponível para a alimentação e acompanhamento das equipes de maneira individualizada. É válido ressaltar ainda, que os indicadores de monitoramento não fizeram parte da composição da nota de avaliação do $P M A Q / A B$ e nem do presente do estudo. 
O programa selecionou inicialmente, 47 indicadores de desempenho, subdivididos em sete áreas estratégicas e classificados segundo a natureza de seu uso. As áreas foram as relacionadas à saúde da mulher, saúde da criança, controle do diabetes mellitus e da hipertensão arterial sistêmica, saúde bucal, produção geral, tuberculose/hanseníase e saúde mental.
O presente estudo incluiu para fins de análise, apenas os indicadores de desempenho das eSF. Dessa maneira, foram considerados os 24 indicadores de desempenho, contemplando todas as áreas avaliadas pelo $P M A Q / A B$, exceto as áreas de tuberculose/ hanseníase e saúde mental (indicadores de monitoramento).

Os dados do $1^{\circ}$ e do $2^{\circ}$ ciclo foram

Quadro 1. Número, indicadores de desempenho das equipes de Saúde da Família, e área estratégica, referentes ao $1^{\circ}$ e $2^{\circ}$ Ciclo do PMAQ-AB

\begin{tabular}{|c|c|c|}
\hline Número & Indicadores de desempenho das equipes de Saúde da Família & Área estratégica \\
\hline 1.1 & Proporção de gestantes cadastradas pela equipe de atenção básica & \multirow{6}{*}{ Saúde da Mulher } \\
\hline 1.2 & Média de atendimentos de pré-natal por gestante cadastrada & \\
\hline 1.3 & Proporção de gestantes que iniciaram o pré-natal no primeiro trimestre & \\
\hline 1.4 & Proporção de gestantes com o pré-natal em dia & \\
\hline 1.5 & Proporção de gestantes com vacina em dia & \\
\hline 1.6 & $\begin{array}{l}\text { Razão entre exames citopatológicos do colo do útero na faixa etária de } \\
15 \text { anos ou mais }\end{array}$ & \\
\hline 2.1 & Média de atendimentos de puericultura & \multirow{6}{*}{ Saúde da Criança } \\
\hline 2.2 & $\begin{array}{l}\text { Proporção de crianças menores de quatro meses com aleitamento ex- } \\
\text { clusivo }\end{array}$ & \\
\hline 2.3 & Proporção de crianças menores de um ano com vacina em dia & \\
\hline 2.4 & Proporção de crianças menores de dois anos pesadas & \\
\hline 2.5 & Média de consultas médicas para menores de um ano & \\
\hline 2.6 & Média de consultas médicas para menores de cinco anos & \\
\hline 3.1 & Proporção de diabéticos cadastrados; & \multirow{4}{*}{$\begin{array}{l}\text { Controle de Diabetes } \\
\text { Mellitus e Hiperten- } \\
\text { são Arterial Sistêmica }\end{array}$} \\
\hline 3.2 & Proporção de hipertensos cadastrados & \\
\hline 3.3 & Média de atendimentos por diabético & \\
\hline 3.4 & Média de atendimentos por hipertenso. & \\
\hline 4.1 & Média da ação coletiva de escovação dental supervisionada; & \multirow{4}{*}{ Saúde Bucal } \\
\hline 4.2 & Cobertura de primeira consulta odontológica programática; & \\
\hline 4.3 & Cobertura de primeira consulta de atendimento odontológico à gestante; & \\
\hline 4.4 & $\begin{array}{l}\text { Razão entre tratamentos concluídos e primeiras consultas odontológicas } \\
\text { programáticas }\end{array}$ & \\
\hline 5.1 & Média de consultas médicas por habitante; & \multirow{4}{*}{ Produção Geral } \\
\hline 5.2 & Proporção de consultas médicas para cuidado continuado/programado & \\
\hline 5.3 & Proporção de consultas médicas de demanda agendada; & \\
\hline 5.4 & Proporção de consultas médicas de demanda imediata & \\
\hline
\end{tabular}

Fonte: Adaptado de Brasil, (2015) ${ }^{5}$. 
comparados estatisticamente. Para isso, os mesmos foram analisados descritivamente por meio de frequências absolutas e percentuais, média, desvio padrão, mediana e percentis P25 e P75 e foram analisados inferencialmente através do teste t-Student pareado ou Wilcoxon para dados pareados. A escolha do teste t-Student ocorreu quando a variável diferença entre os ciclos teve distribuição normal e de Wilcoxon para dados pareados quando a hipótese de normalidade foi rejeitada. $A$ verificação da hipótese de normalidade foi realizada por meio do teste de Kolmogorov-Smirnov.

O programa utilizado para elaboração dos cálculos estatísticos foi o SPSS versão 23. A margem de erro utilizada na decisão dos testes estatísticos foi de 5,0\%.

\section{RESULTADOS}

Ocorreram diferenças significativas

Tabela 1. Comparação dos indicadores de desempenho por ciclo do PMAQ-AB na região do Nordeste do Brasil

\begin{tabular}{|c|c|c|c|c|c|c|}
\hline \multirow[b]{2}{*}{ Indicador } & \multicolumn{2}{|r|}{$1^{\circ} \mathrm{Ciclo}$} & \multicolumn{2}{|c|}{$2^{\circ}$ Ciclo } & \multirow{2}{*}{\begin{tabular}{|c|} 
Média \\
$\mathrm{C} 2-\mathrm{C} 1$ \\
\end{tabular}} & \multirow[b]{2}{*}{ Valor $p$} \\
\hline & Média \pm DP & $\begin{array}{c}\mathrm{P} 50 \\
(\mathrm{P} 25 ; \mathrm{P} 75)\end{array}$ & Média \pm DP & $\begin{array}{c}\mathrm{P} 50 \\
(\mathrm{P} 25 ; \mathrm{P} 75)\end{array}$ & & \\
\hline \multicolumn{7}{|c|}{ Saúde da Mulher } \\
\hline 1.1 & $52,90 \pm 18,77$ & $50,99(39,94 ; 64,20)$ & $46,84 \pm 18,10$ & $45,08(34,29 ; 57,82)$ & $-6,06$ & $\mathrm{p}^{(1)}<0,001^{*}$ \\
\hline 1.2 & $9,06 \pm 2,58$ & $9,04(7,39 ; 10,94)$ & $7,96 \pm 2,99$ & $8,24(6,05 ; 10,09)$ & $-1,10$ & $\mathrm{p}^{(1)}<0,001^{*}$ \\
\hline 1.3 & $84,14 \pm 16,23$ & $88,24(76,80 ; 96,00)$ & $84,00 \pm 14,81$ & $87,58(77,11 ; 94,87)$ & $-0,14$ & $p^{(1)}=0,098^{*}$ \\
\hline 1.4 & $94,32 \pm 9,91$ & $99,10(91,67 ; 100,00)$ & $93,68 \pm 8,87$ & $96,83(91,11 ; 100,00)$ & $-0,64$ & $\mathrm{p}^{(1)}<0,001^{*}$ \\
\hline 1.5 & $95,94 \pm 8,70$ & $100,00(94,74 ; 100,00)$ & $95,60 \pm 7,59$ & $98,72(94,06 ; 100,00)$ & $-0,34$ & $\mathrm{p}^{(1)}<0,001^{*}$ \\
\hline \multirow[t]{2}{*}{1.6} & $37,10 \pm 42,57$ & $12,19(0,00 ; 94,10)$ & $0,07 \pm 0,08$ & $0,03(0,00 ; 0,12)$ & $-37,03$ & $\mathrm{p}^{(1)}<0,001^{*}$ \\
\hline & \multicolumn{4}{|c|}{ Saúde da Criança } & & \\
\hline 2.1 & $6,11 \pm 5,00$ & $4,96(2,55 ; 8,56)$ & $5,06 \pm 4,85$ & $3,82(1,08 ; 7,63)$ & $-1,05$ & $p^{(1)}<0,001^{*}$ \\
\hline 2.2 & $70,02 \pm 22,61$ & $73,29(60,00 ; 85,71)$ & $65,70 \pm 22,13$ & $68,81(55,68 ; 80,56)$ & $-4,32$ & $\mathrm{p}^{(1)}<0,001^{*}$ \\
\hline 2.3 & $95,44 \pm 8,77$ & $99,26(94,12 ; 100,00)$ & $96,04 \pm 6,56$ & $98,51(94,85 ; 100,00)$ & 0,60 & $p^{(1)}=0,233$ \\
\hline 2.4 & $89,91 \pm 16,68$ & $96,70(87,76 ; 100,00)$ & $88,63 \pm 17,15$ & $95,13(85,67 ; 99,31)$ & $-1,28$ & $\mathrm{p}^{(1)}<0,001^{*}$ \\
\hline 2.5 & $3,03 \pm 3,05$ & $2,16(1,03 ; 3,93)$ & $3,10 \pm 2,99$ & $2,20(1,12 ; 4,07)$ & 0,07 & $p^{(1)}=0,191$ \\
\hline \multirow[t]{2}{*}{2.6} & $1,72 \pm 2,06$ & $1,22(0,00 ; 2,54)$ & $1,45 \pm 1,85$ & $0,89(0,00 ; 2,25)$ & $-4,66$ & $\mathrm{p}^{(2)}<0,001^{*}$ \\
\hline & \multicolumn{4}{|c|}{ Controle de Diabetes Mellitus e Hipertensão Arterial Sistêmica } & & \\
\hline 3.1 & $51,29 \pm 37,82$ & $63,74(0,00 ; 81,88)$ & $53,47 \pm 37,82$ & $65,13(0,00 ; 83,82)$ & 2,18 & $p^{(2)<0,001^{*}}$ \\
\hline 3.2 & $72,34 \pm 15,63$ & $71,20(59,94 ; 83,87)$ & $72,87 \pm 15,46$ & $71,12(60,69 ; 85,07)$ & 0,53 & $p^{(2)}=0,071$ \\
\hline 3.3 & $6,03 \pm 3,88$ & $5,26(3,17 ; 8,12)$ & $6,20 \pm 3,90$ & $5,37(3,49 ; 8,07)$ & 0,17 & $p^{(2)}=0,510$ \\
\hline \multirow[t]{2}{*}{3.4} & $4,73 \pm 3,24$ & $4,05(2,32 ; 6,48)$ & $4,48 \pm 2,96$ & $3,73(2,47 ; 5,82)$ & $-0,25$ & $\mathrm{p}^{(2)}<0,001^{*}$ \\
\hline & \multicolumn{4}{|c|}{ Saúde Bucal } & & \\
\hline 4.1 & $3,27 \pm 6,26$ & $0,98(0,00 ; 3,89)$ & $2,38 \pm 4,33$ & $0,75(0,00 ; 2,88)$ & $-0,89$ & $\mathrm{p}^{(1)}<0,001^{*}$ \\
\hline 4.2 & $1,29 \pm 1,62$ & $0,87(0,00 ; 1,88)$ & $1,20 \pm 1,40$ & $0,86(0,08 ; 1,76)$ & $-0,09$ & $\mathrm{p}^{(1)}<0,001^{*}$ \\
\hline 4.3 & $19,57 \pm 22,25$ & $12,24(1,85 ; 29,12)$ & $16,43 \pm 18,48$ & $10,74(2,22 ; 23,61)$ & $-3,14$ & $\mathrm{p}^{(1)}<0,001^{*}$ \\
\hline \multirow[t]{2}{*}{4.4} & $33,04 \pm 34,74$ & $22,64(7,98 ; 46,79)$ & $30,39 \pm 30,02$ & $21,75(8,12 ; 44,08)$ & $-2,65$ & $p^{(1)}=0,089$ \\
\hline & \multicolumn{4}{|c|}{ Produção Geral } & & \\
\hline 5.1 & $1,28 \pm 0,75$ & $1,16(0,78 ; 1,63)$ & $1,53 \pm 0,91$ & $1,34(0,90 ; 1,97)$ & 0,25 & $\mathrm{p}^{(1)}<0,001^{*}$ \\
\hline 5.2 & $20,83 \pm 18,97$ & $18,05(5,26 ; 30,26)$ & $21,74 \pm 19,31$ & $18,79(6,22 ; 31,39)$ & 0,91 & $p^{(1)}=0,001^{*}$ \\
\hline 5.3 & $50,86 \pm 28,03$ & $55,03(29,54 ; 72,82)$ & $50,38 \pm 26,51$ & $53,13(31,37 ; 70,64)$ & $-0,48$ & $p^{(1)}=0,125$ \\
\hline 5.4 & $28,15 \pm 27,49$ & $18,73(6,86 ; 41,74)$ & $27,88 \pm 26,05$ & $20,07(7,68 ; 41,17)$ & $-0,27$ & $p^{(1)}=0,718$ \\
\hline
\end{tabular}


em relação aos indicadores de desempenho da saúde da mulher entre $\circ 1^{\circ}$ e o $2^{\circ}$ ciclo do PMAQ/AB (Tabela 1). Para as mulheres, a maioria dos indicadores do $1^{\circ}$ ciclo foram maiores que no $2^{\circ}$ ciclo. Foi observada uma maior diferença de desempenho entre o $1^{\circ} \mathrm{e}$ $2^{\circ}$ ciclo do indicador razão entre os exames citopatológicos do colo do útero na faixa etária de 15 anos ou mais, média de 37,10 e 0,07 respectivamente.

Tabela 2. Média das variações entre ciclos dos indicadores de desempenho por estado na região Nordeste

\begin{tabular}{|c|c|c|c|c|c|c|c|c|c|}
\hline \multirow[b]{2}{*}{ Indicador } & \multicolumn{8}{|c|}{ Estado } & \multirow[b]{2}{*}{ SE } \\
\hline & $\mathrm{AL}$ & $\mathrm{BA}$ & CE & MA & PB & PE & $\mathrm{PI}$ & RGN & \\
\hline \multicolumn{10}{|c|}{ Saúde da Mulher } \\
\hline 1.1 & $-5,60$ & $-7,31$ & $-5,03$ & $-10,93$ & $-5,44$ & $-6,89$ & $-3,02$ & $-4,58$ & $-4,49$ \\
\hline 1.2 & $-0,93$ & $-1,10$ & $-1,98$ & 0,10 & $-0,96$ & $-0,96$ & $-0,29$ & $-0,89$ & $-1,29$ \\
\hline 1.3 & $-0,01$ & $-0,52$ & $-0,96$ & $-3,03$ & 0,71 & 0,39 & 1,00 & 0,01 & 0,51 \\
\hline 1.4 & $-1,03$ & $-1,20$ & $-1,03$ & $-0,04$ & $-0,15$ & $-0,27$ & 0,51 & 0,05 & $-0,71$ \\
\hline 1.5 & $-0,96$ & $-1,35$ & $-0,79$ & $-0,24$ & 0,76 & 0,31 & 0,50 & 0,99 & 0,05 \\
\hline 1.6 & $-58,89$ & $-42,27$ & $-20,20$ & $-63,98$ & $-30,17$ & $-32,79$ & $-48,23$ & $-35,46$ & $-35,94$ \\
\hline \multicolumn{10}{|c|}{ Saúde da Criança } \\
\hline 2.1 & $-1,59$ & $-1,45$ & $-0,51$ & $-0,59$ & $-0,73$ & $-1,95$ & 0,92 & $-1,04$ & $-0,42$ \\
\hline 2.2 & $-11,32$ & $-4,14$ & $-5,01$ & $-3,56$ & $-4,04$ & $-5,48$ & 16,22 & $-14,87$ & $-4,58$ \\
\hline 2.3 & 0,80 & 0,48 & $-1,20$ & 0,22 & 1,34 & 1,06 & 0,75 & 2,19 & 1,13 \\
\hline 2.4 & $-2,51$ & $-1,39$ & $-3,26$ & $-5,49$ & $-1,55$ & 0,15 & $-0,63$ & 1,52 & 0,34 \\
\hline 2.5 & 0,80 & $-0,10$ & 0,00 & $-0,82$ & 0,52 & 0,37 & $-0,56$ & $-0,08$ & $-0,41$ \\
\hline 2.6 & $-0,20$ & $-0,33$ & $-0,57$ & $-0,55$ & 0,05 & $-0,26$ & 0,26 & $-0,45$ & $-0,09$ \\
\hline \multicolumn{10}{|c|}{ Controle de DM e HAS } \\
\hline 3.1 & $-3,53$ & 2,36 & 4,21 & $-0,89$ & 0,98 & 2,12 & 14,67 & $-7,05$ & 3,14 \\
\hline 3.2 & 0,41 & $-0,10$ & 1,80 & $-2,18$ & $-0,35$ & 0,51 & 2,93 & 0,71 & 1,32 \\
\hline 3.3 & 0,32 & 0,29 & $-0,01$ & $-1,12$ & $-0,20$ & 1,05 & $-0,71$ & $-0,33$ & 0,28 \\
\hline 3.4 & $-0,15$ & $-0,07$ & $-0,85$ & $-0,99$ & $-0,65$ & 0,65 & $-0,66$ & $-0,72$ & 0,43 \\
\hline \multicolumn{10}{|c|}{ Saúde Bucal } \\
\hline 4.1 & $-1,26$ & $-0,17$ & $-2,37$ & $-0,92$ & $-0,63$ & $-0,69$ & $-1,63$ & $-0,68$ & $-0,44$ \\
\hline 4.2 & $-0,25$ & $-0,09$ & $-0,09$ & 0,14 & $-0,36$ & 0,02 & 0,35 & $-0,22$ & $-0,26$ \\
\hline 4.3 & $-0,96$ & $-2,01$ & $-4,00$ & $-7,04$ & $-3,85$ & $-2,47$ & $-3,04$ & $-5,12$ & $-4,08$ \\
\hline 4.4 & $-3,29$ & $-2,96$ & $-3,66$ & 4,06 & $-4,10$ & $-2,70$ & 1,47 & $-2,66$ & $-0,05$ \\
\hline \multicolumn{10}{|c|}{ Produção Geral } \\
\hline 5.1 & 0,42 & 0,23 & 0,27 & $-0,18$ & 0,33 & 0,34 & $-0,04$ & 0,18 & 0,41 \\
\hline 5.2 & 1,76 & 0,30 & $-0,20$ & $-1,89$ & $-0,13$ & 3,37 & 1,62 & 1,96 & $-0,26$ \\
\hline 5.3 & $-2,84$ & 0,32 & 1,37 & 0,72 & $-0,84$ & $-3,10$ & $-1,40$ & 0,73 & 0,62 \\
\hline 5.4 & 1,08 & $-0,62$ & $-0,80$ & 1,18 & 0,97 & $-0,09$ & $-0,21$ & $-2,26$ & 0,43 \\
\hline
\end{tabular}


Sobre os indicadores da saúde da criança, observou-se um desempenho meIhor do indicador de proporção de crianças menores de um ano com vacina em dia, no $2^{\circ}$ ciclo do programa, quando comparado com o $1^{\circ}$ ciclo. Os indicadores que apresentaram um pior desempenho foram os relacionados às consultas médicas para menores de cinco anos e proporção de crianças menores de quatro meses com aleitamento exclusivo.

Foi possível identificar também um aumento no número de diabéticos cadastrados no $2^{\circ}$ ciclo na Região do Nordeste, bem como o número de atendimentos para esse segmento da população. No entanto, quando se trata dos usuários hipertensos, apesar do número de cadastramento ter sido maior no $2^{\circ}$ ciclo, o número de atendimentos foi significativamente menor.

Em relação aos indicadores de saúde bucal foi possível afirmar que todos tiveram um desempenho menor no $2^{\circ}$ ciclo, sendo que o indicador de primeira consulta de atendimento odontológico à gestante e de razão entre tratamentos concluídos e primeiras consultas odontológicas programáticas, apresentaram desempenhos significativamente menores que os demais indicadores.

Dos indicadores de produção geral, verificou-se o melhor desempenho no indicador proporção de consultas médicas para cuidado continuado/programado, quando comparado entre os ciclos. O indicador proporção de consultas médicas de demanda agendada foi significativamente menor no $2^{\circ}$ ciclo.

A Tabela 2 demostra as médias das variações entre os dois ciclos de cada um dos indicadores por estado. Dentre as áreas avaliadas pelo $\mathrm{PMAQ} / \mathrm{AB}$ destacaram-se as variações dos indicadores de saúde referentes à saúde da mulher. Em todas os estados do Nordeste o indicador proporção de gestantes cadastradas pela eSF e a razão entre os exames citopatológicos do colo do útero na faixa etária de 15 anos ou mais, foram menores no $2^{\circ}$ ciclo.

\section{DISCUSSÃO}

Comparando os resultados dos indicadores de desempenho entre o $1^{\circ}$ Ciclo e o $2^{\circ}$ Ciclo do PMAQ-AB no Nordeste do Brasil, foi possível observar que apenas os indicadores de proporção de pacientes diabéticos cadastrados, média de consultas médicas/habitante, proporção de consultas médicas para cuidado médico continuado/prolongado foram aprimorados durante a vigência do programa. Apesar desses resultados positivos, esse é um cenário que apresentou pouca melhoria em se tratando da potencialidade do PMAQ-AB. De forma geral, a maioria dos indicadores de saúde considerados para a avaliação do programa, apresentou menor desempenho no $2^{\circ}$ ciclo, dentre eles destacou-se os referentes à saúde da mulher e da criança.

Quando se tratou dos indicadores que tiveram melhor desempenho, a proporção de pacientes diabéticos castrados pela eSF se destacou. O diabetes mellitus é uma condição que lidera como causa de cegueira, doença renal, amputação, além de expor a um aumento de mortalidade, principalmente por eventos cardiovasculares ${ }^{14}$. Assim, a atenção integral aos diabéticos é essencial na atenção básica, no entanto, apesar do aumento da pro- 
porção de pacientes diabéticos cadastrados o presente estudo revelou a necessidade de melhorias nos indicadores relacionados às doenças crônicas.

Um estudo comparativo entre $01^{\circ} \mathrm{e} 2^{\circ}$ Ciclo do PMAQ-AB, identificou que a Região Norte e Nordeste registrou o menor número de Unidades Básicas de Saúde com estrutura física adequada para prestação de serviços voltados aos usuários diabéticos ${ }^{14}$. Outras pesquisas também reforçam a precariedade nos serviços ofertados nessas regiões do país ${ }^{15,16}$ Tal informação pode estar contribuindo para apontar, de forma negativa, o dinamismo do cuidado integral prestado ao portador de diabetes mellitus na Região Nordeste, estendendo-se também para outras condições crônicas, como identificado nos indicadores avaliados, especialmente o que se refere ao atendimento aos pacientes hipertensos.

Com relação à assistência médica, ocorreu um aumento de acesso às consultas médicas ao longo dos últimos anos, especialmente no Nordeste ${ }^{17}$. Segundo Gonçalves et al. ${ }^{18}$, entre os anos de 2012 a 2015, verificou-se uma redução de hospitalizações por condições sensíveis à atenção primária, atribuído a implantação do Programa Mais Médicos na Região do Nordeste. O Programa Mais Médicos tem como objetivo principal a formação em saúde e a provisão de médicos em regiões prioritárias para o SUS. Dos 14.465 médicos que iniciaram suas atividades entre os anos de 2013 a 2014, 33,4\% foram para o nordeste do país, desses $83 \%$ foram alocados em municípios que enfrentam alguma situação de vulnerabilidade ${ }^{19}$. Sugere-se, portanto, que o maior número de profissionais médicos inseridos na ESF nesta região do país, ao longo dos anos, tenha contribuído com um maior número de consultas médicas e direcionadas para o cuidado continuado, conforme demonstram os achados presentes.

Dentre os indicadores com piores desempenhos, evidencia-se a proporção de gestantes cadastradas pela eSF e a média de atendimentos de pré-natal por gestante cadastrada no $1^{\circ}$ e $2^{\circ}$ ciclo do programa. Os baixos indicadores de atenção à gestante no Brasil é um problema já identificado em estudos anteriores ${ }^{20,21}$. Segundo Tomasi et al. ${ }^{20}$ os indicadores de qualidade da atenção pré-natal no Brasil do $1^{\circ}$ ciclo do PMAQ-AB em toda as regiões brasileiras revelaram que apenas $15 \%$ de 6.125 mulheres entrevistadas foram adequadamente assistidas pela eSF, destacando-se a baixa prática dos exames físicos e orientações preconizadas durante as consultas. O mesmo estudo já apontava que a Região Nordeste, devido as suas características de desigualdades sociais e econômicas, apresentava um baixo desempenho em critérios importantes, aquém da oferta de um pré-natal de qualidade.

Frente a essa informação, sugere-se que algumas estratégias embasadas nos Manuais do Ministério da Saúde possam ser aplicadas para facilitar a captação precoce de gestantes na unidade. A captação precoce das gestantes pode ser por meio de visitas domiciliares a serem realizadas, preferencialmente, pelos agentes comunitários de saúde, consistindo na busca ativa por mulheres que apresentam amenorreia há mais de quinze 
dias e na orientação quanto à importância do pré-natal ${ }^{22}$. Outra possibilidade de captação precoce da gestante na comunidade é o oferecimento do teste imunológico para gravidez (TIG), que pode ser realizado pelo profissional de saúde da unidade básica. Para facilitar o acesso pode-se realizar um atendimento diferenciado para as gestantes mantendo agenda aberta sem necessidade de marcar consulta e que o profissional tenha disponibilidade para ouvir a gestante, tirar suas dúvidas e prestar os esclarecimentos necessários, mesmo que necessite dispender mais tempo no acolhimento $^{23}$.

Ainda entre os baixos indicadores referentes à saúde da mulher identificados nos achados presentes, destaca-se a diminuição expressiva da razão entre os exames citopatológicos do colo do útero na faixa etária de 15 anos ou mais. O exame citopatológico consiste em uma técnica de detecção precoce do câncer de colo de uterino, a partir do rastreamento de lesões que podem se tornar invasivas e malignas, sendo o procedimento mais efetivo em programas de rastreamento desse tipo de câncer ${ }^{24}$. Algumas estratégias podem contribuir para a adesão das mulheres ao exame preventivo do câncer de colo uterino, como a oferta de horários diferenciados e flexíveis para a realização do exame. Os profissionais de saúde devem interagir com as mulheres a fim de individualizar a assistência prestada.

No entanto, o Nordeste apresenta baixa frequência na periodicidade do exame, sendo ainda menor entre as de pouco nível de escolaridade e mulheres de baixo estrato socioeconômico ${ }^{25}$. No presente estudo, as regiões que revelaram diferenças importantes do $1^{\circ}$ e para o $2^{\circ}$ Ciclo do PMAQ-AB foram os Estados do Maranhão, Alagoas e Piauí.

O Nordeste tem o maior número de pessoas em situações de pobreza e extrema miséria quando comparado com as outras regiões brasileiras, condição que vem aumentando consideravelmente nos últimos anos ${ }^{26}$. O Estado do Maranhão é o estado do país que apresenta o maior número de pessoas em situação de pobreza, o número de pessoas que vivem com $R \$ 406$ reais por mês corresponde mais que a metade da população local $(54,1 \%)$, seguido do Estado de Alagoas e Piauí $^{26}$. Ainda sobre rendimentos, sabe-se que entre os trabalhadores ocupados, o Maranhão apresenta o menor rendimento médio salarial ( $R$ \$ 1.170,00), seguido do Estado do Piauí ( $R$ \$ $1.233,00)$ e Alagoas $(R \$ 1.309,00)^{26}$.

Da mesma maneira que as precárias condições de vida afetam a saúde da mulher e o acesso aos serviços de saúde, a saúde da criança também sofre com as condições de vida da família. Comparando os indicadores do $1^{\circ}$ e do $2^{\circ}$ Ciclo do PMAQ/AB em relação à saúde da criança, chamou atenção a significativa diminuição do indicador de proporção de crianças menores de quatro meses com aleitamento materno exclusivo.

$\mathrm{O}$ aleitamento materno exclusivo (AME) até os seis primeiros meses de vida e a sua manutenção complementada por outros alimentos, até os dois anos de vida, é um dos métodos que mais contribui para a manutenção da saúde do bebê e auxilia na redução da morbimortalidade infantil ${ }^{27}$. No Nordeste, a desvalorização da prática da AME já foi apontada como consequência do desconhecimento da sua importância entre a comunidade ${ }^{28}$ e devido à escolaridade da 
mãe ${ }^{29}$. Em entrevistas com 354 puérperas do Estado do Ceará foi identificado a porcentagem de mães que ofereciam AME no primeiro, quarto e sexto mês, sendo de $39,2 \%(n=109)$, $7,6 \%(n=21)$ e $1,1 \%(n=3)$, respectivamente. Os mesmos autores ainda encontraram que $57,6 \%$ das mulheres que não realizaram o AME, não receberam orientações sobre essa prática durante o pré-natal e a maioria tinham baixa escolaridade ${ }^{29}$. Nessa perspectiva, se faz necessário discutir estratégias efetivas que aumentem o tempo de amamentação materna exclusiva e reorganizem o cuidado prestado pela atenção básica às mães e aos bebês.

Por fim, salientamos que este estudo é ecológico e, portanto, apresenta limitações inerentes. A pesquisa foi baseada em um banco de dados secundários, cuja precisão não pode ser avaliada, existindo a possibilidade do viés de informação. Além disso, o número de equipes participantes dos ciclos do PMAQ-AB foi controlado pelos gestores de saúde e teve a sua adesão voluntária, o que pode ter levado a uma seleção de equipes mais comprometidas, esse viés de seleção pode superestimar os resultados encontrados. É necessário apontar ainda, que existe a possibilidade das eSF não participantes do programa apresentarem desempenho ainda menores que as eSF contratualizadas no $P M A Q / A B$, sugere-se assim que essa hipótese seja investigada em outros estudos. No entanto, apesar das limitações identificadas, os achados revelaram um ce- nário preocupante, de baixo desempenho de indicadores importantes de saúde da população no Nordeste do Brasil.

Esperava-se que o PMAQ-AB contribuísse para que os serviços se organizassem de maneira a reconhecer as necessidades da população e reestruturassem o processo de trabalho contando com o apoio de incentivos financeiros. Porém, apesar do PMAQ-AB ser uma ferramenta importante para a condução das ações das equipes de saúde e dos gestores, as deficiências de comunicação entre esses atores, o engajamento dos profissionais e o entendimento dessa política de avaliação, ainda são barreiras que fragilizam as mudanças propostas pelo PMAQ-AB ${ }^{30}$. Ressalta-se ainda, que o modo como os relatórios gerados pelo PMAQ-AB, após os ciclos do programa, foi utilizado pela gestão local e pelas eSF para aprimoramento do processo de trabalho, é um aspecto pouco elucidado na literatura, sendo necessário estudos adicionais sobre esta temática. Por fim, destaca-se que o PMAQ-AB ou outros programas desenvolvidos para avaliação de desempenhos dos indicadores da atenção básica, por si só, não serão capazes de ser um movimento de transformações visando à qualidade do cuidado, necessitando também de articulações intersetoriais que operem de forma concomitante com os determinantes sociais que inviabilizam o acesso e a qualidade dos serviços de saúde. 


\section{REFERÊNCIAS}

1. Giovanella, L. Atenção básica ou atenção primária à saúde? Cad. Saúde Pública 2018, 34(8)e:00029818.

2. Facchini LA, Tomasi E, Dilélio AS. Qualidade da Atenção Primária à Saúde no Brasil: avanços, desafios e perspectivas. Saúde Debate. 2018; 42(1):208-223.

3. Furtado JP, Campos GWS, Oda WY, Onocko CR. Planejamento e Avaliação em Saúde: entre antagonismo e colaboração. Cad. Saúde Pública. 2018; 34(7) :e00087917.

4. Brasil. Ministério da Saúde. Avaliação da Melhoria da Estratégia de Saúde da Família. Guia de Implantação municipal AMQ. Secretaria de Atenção à Saúde. Brasília, 2006.

5. Brasil. Ministério da Saúde. Secretaria de Atenção à Saúde. Departamento de Atenção Básica. Qualificação dos Indicadores do Manual Instrutivo para as equipes de Atenção Básica (Saúde da Família, Saúde Bucal e Equipes Parametrizadas) e NASF [recurso eletrônico] / Ministério da Saúde, Secretaria de Atenção à Saúde, Departamento de Atenção Básica. - Brasília : Ministério da Saúde, 2015.

6. Neves RG, Duro SMS, Muñiz J, Castro TRP, Facchini LA, Tomasi E. Estrutura das unidades básicas de saúde para atenção às pessoas com diabetes: Ciclos I e II do Programa Nacional de Melhoria do Acesso e da Qualidade. Cad Saúde Pública. 2018; 34 (4):e00072317.

7. Protasio APL, Gomes LB, Machado LS, Valenca, ANG. Satisfação do usuário da Atenção Básica em Saúde por regiões do Brasil: $1^{\circ}$ ciclo de avaliação externa do PMAQ-AB. Ciênc. Saúde Coletiva. 2017; 22(6):1829-1844.

8. Carvalho BR; Ferreira, JBB, Fausto MCR, Forster AC. Avaliação do acesso às unidades de atenção primária em municípios brasileiros de pequeno porte. Cad. Saúde Colet. 2018; 26(4):462-469.

9. Casotti E, Contarato PC, Fonseca ABM, Borges PKO, Baldani MH. Atenção em Saúde Bucal no Brasil: uma análise a partir da Avaliação Externa do PMAQ-AB. Saúde Debate. 2014; 38(spe):140-157.

10. Cruz MM, Souza RBC, Torres RMC, Abreu DMF, Reis AC, Gonçalves AL. Usos do planejamento e autoavaliação nos processos de trabalho das equipes de Saúde da Família na Atenção Básica. Saúde Debate. 2014; 38(spe):124139.
11. Brocardo D, Andrade CLT, Fausto MCR, Lima SML. Núcleo de Apoio à Saúde da Família (Nasf): panorama nacional a partir de dados do PMAQ. Saúde debate. 2018; 42(spe1):130-144.

12. Brasil. Ministério da Saúde. Banco de dados do Sistema Único de Saúde-DATASUS. (Acessado 2020).

13. Brasil. Ministério da Saúde. Portaria $n^{\circ} .1 .412$, de 10 de julho de 2013. Sistema de Informação em Saúde para Atenção Básica- SISAB, Brasília (DF); 2013.

14. Moreira RO, Papelbaum M, Appolinario JC, Matos AG, Coutinho WF, Meirelles RMR et al. Diabetes mellitus e depressão: uma revisão sistemática. Arq Bras Endocrinol Metab. 2003; 47(1):19-29.

15. Facchini LA, Piccini RX, Tomasi E, Thumé E, Silveira DS, Siqueira FV, et al. Desempenho do PSF no Sul e no Nordeste do Brasil: avaliação institucional e epidemiológica da atenção básica à saúde. Ciênc Saúde Coletiva 2006; 11:669-681.

16. Tomasi E, Cesar MADC, Neves RG, Schmidt PRC, Thumé E, Silveira DS, et al. Diabetes care in Brazil: Program to Improve Primary Care Access and Quality-PMAQ. J Ambul Care Manage 2017; 40 (Suppl 2):12-23.

17. Silva PSC, Boing AF, Peres KG. Redução das desigualdades no uso de consultas médicas no Brasil: análise das regiões Nordeste e Sudeste entre 2003 e 2008. Rev Bras Epidemiol. 2015;18(1):248-261.

18. Gonçalves RF, Sousa IMC, Tanaka OY, Santos CR, Brito-Silva KS, Lara $X$ et al. Programa Mais Médicos no Nordeste: avaliação das internações por condições sensíveis à Atenção Primária à Saúde. Ciênc Saúde Coletiva. 2016; 21(9):2815-2824.

19. Brasil. Ministério da Saúde. Mais Médicos. Sistema de Gerenciamento de Programas: consultar médicos. 2015 [acessado 2018 jul 4].

20. Tomasi E, Fernandes PAA, Fischer T, Siqueira FCV, Silveira DS, Thumé E, et al. Qualidade da atenção pré-natal na rede básica de saúde do Brasil: indicadores e desigualdades sociais. Cad. de Saúde Pública. 2017; 33(3): e00195815. 
21. Gonzaga ICA, Santos SLD, Silva ARV, Campelo V. Atenção pré-natal e fatores de risco associados à prematuridade e baixo peso ao nascer em capital do nordeste brasileiro. Ciênc. saúde coletiva. 2016, 21(6):1965-1974.

22. Brasil. Ministério da Saúde. Secretaria de Atenção à Saúde. Departamento de Ações Programáticas Estratégicas. Área Técnica de Saúde da Mulher. Pré-natal e puerpério: atenção qualificada e humanizada: manual técnico. Brasília: Ministério da Saúde; 2005.

23. Duncan BB, Schmidt MI, Giugliani ERJ. Medicina Ambulatorial: Condutas de Atenção Primária Baseada em Evidências. 3. ed. Porto Alegre: Artmed, 2006.

24. Instituto Nacional de Câncer. Estimativa 2010: incidência de câncer no Brasil. Rio de Janeiro: Instituto Nacional de Câncer; 2011.

25. Correa MS, Silveira DS, Siqueira FV, Facchini LA, Piccini RX, Thumé E, et al. Cobertura e adequação do exame citopatológico de colo uterino em estados das regiões Sul e Nordeste do Brasil. Cad Saúde Pública. 2012; 28(12):2257-2266.

26. Síntese de indicadores sociais: uma análise das condições de vida da população brasileira: 2018/IBGE, Coordenação de População e Indicadores Sociais. - Rio de Janeiro: IBGE, 2018.
27. Brasil. Saúde da Criança: Nutrição Infantil: Aleitamento Materno e Alimentação Complementar. Brasília (DF): Ministério da Saúde; 2009.

28. Leal AB, Sousa AF, Florentino EC, Silva LR, Menezes CC. Perfil do aleitamento materno exclusivo e fatores determinantes do desmame precoce em município do semi-árido da Região Nordeste. Rev Bras Pesq Saúde. 2014; 16(3):84-91

29. Cavalcanti SH, Caminha MFC, Figueiroa JN, Serva VMSBD, Cruz RSBLC, Lira PIC et al. Fatores associados à prática do aleitamento materno exclusivo por pelo menos seis meses no estado de Pernambuco. Rev. bras. Epidemiol. 2015;18(1):208-219.

30. Flôres GMS, Weigelt LD, Rezende MS, Telles R, Krug SBF. Gestão pública no SUS: considerações acerca do PMAQ-AB. Saúde debate. 2018; 42(116):237-247.

\section{CORRESPONDÊNCIA}

Inara Pereira da Cunha

Avenida Limeira, 901 - CEP 13.414-903,

Piracicaba, SP.

E-mail: inara-pereira@hotmail.com 